\title{
Application of Decision Tree in the Prediction of Periventricular Leukomalacia (PVL) Occurrence in Neonates After Heart Surgery
}

\author{
Ali Jalali ${ }^{1}$, Daniel J. Licht ${ }^{2}$ and C. Nataraj ${ }^{3}$
}

\begin{abstract}
This paper is concerned with the prediction of the occurrence of periventricular leukomalacia (PVL) that occurs in neonates after heart surgery. The data which is collected over a period of 12 hours after cardiac surgery contains vital measurements as well as blood gas measurements with different resolutions. Vital data measured using near-inferred spectroscopy (NIRS) at the sampling rate of $0.25 \mathrm{~Hz}$ and blood gas measurement up to 12 times with irregular time intervals for 35 patients collected at Children's Hospital of Philadelphia (CHOP) are used for this study. Features derived from the data include statistical moments (mean, variance, skewness and kurtosis), trend and minimum and maximum values of the vital data and rate of change, time weighted mean and a custom defined out of range index (ORI) for the blood gas data. A decision tree is developed for the vital data in order to identify the most important vital measurements. In addition, a decision tree is developed for blood gas data to find important factors for the prediction of PVL occurrence. Results show that in the blood gas data, maximum rate of change of concentration of bicarbonate ions in blood $\left(\mathrm{HCO}_{3}\right)$ and minimum rate of change of partial pressure of dissolved $\mathrm{CO}_{2}$ in the blood $\left(\mathrm{PaCO}_{2}\right)$ are the two most important factors for prediction of the PVL. Also important are the kurtosis of heart rate and hemoglobin values.
\end{abstract}

\section{INTRODUCTION}

Periventricular leukomalacia (PVL) is a neonatal brain injury. The PVL condition causes damage in the ventricles of the brain [1]. Generally motor control problems or other neuro-developmental problems including cerebral palsy or epilepsy are common in PVL patients [2], [3]. Recent research has shown that PVL is very common in neonates before and after cardiac surgery [4]-[6], and hence there is a growing interest among clinical researchers to attempt to understand the physiology and pathology of PVL, develop clinical protocols for the prediction and prevention of PVL and also to predict the outcomes of individual patients suffering from PVL [7], [8].

Despite advancement in research in the field of PVL, there are no treatments currently prescribed for PVL. This is due to fact that the origin of PVL and its physiology still remain to be clearly understood. Consequently, all treatment strategies are based in response to pathologies that develop as a consequence of the PVL. In view of the fact that white matter injury in the ventricular parts of the brain can cause

\footnotetext{
${ }^{1}$ A. Jalali is with Department of Mechanical Engineering, Villanova University, 800 E. Lancaster Ave., Villanova, PA, USA email: ali.jalali@villanova.edu

${ }^{2}$ D. J. Licht is Director of Neurovascular Imaging Lab, Children's Hospital of Philadelphia, Philadelphia, PA, USA email : licht@email. chop. edu

${ }^{3}$ C. Nataraj is Mr. and Mrs. Robert F. Moritz, Sr. Endowed Chair Professor in Engineered Systems, Villanova University, Villanova, PA, USA natarajevillanova.edu
}

a variety of deficits and side effects, constant monitoring of PVL infants is necessary to determine the severity of their conditions [9].

Computational intelligence (CI) techniques attract more and more attention from researchers in the biomedical field as a result of their superior performance in comparison with traditional stochastic approaches in prediction, modeling and classification of biomedical signals [10]-[14]. Data mining facilitates data exploration using analysis methods with sophisticated algorithms in order to discover unknown patterns. The CI techniques include data mining algorithms and techniques such as decision tree (DT) [14]-[16], artificial neural networks (ANNs) [17], support vector machine (SVM), and adaptive neuro-fuzzy inference system (ANFIS) [18].

The main advantages of the decision tree approach are the ability to discover rules hidden in the dataset, the ability to handle both continuous and categorical output variables, and construction of easily interpretable classification rules. Moreover, DT algorithms produce noise robust models and rules because they are constructed based on maximizing the information gain. Also DT algorithms produce reliable and effective results with high accuracy and could handle missing data and are especially appropriate to support decisionmaking processes in medicine.

In this study, we explore Decision Tree algorithms to discover rules from the collected data in order to help clinicians predict the occurrence of the PVL. The aim is to identify the most important measurements and derived features based on the extracted classification rules with the expectation that the rules will enable better management of the patients.

\section{MATERIALS AND METHODS}

\section{A. Data Collection}

Data from 35 patients after neonatal cardiac surgery were collected according to a pre-specified protocol at the Children's Hospital of Philadelphia (CHOP). Subjects of this study are limited to two cases of congenital heart disease, hypoplastic left heart syndrome (HLHS) and transposition of great arteries (TGA), accounting for the fact that these two diseases are considered to have the highest likelihood of PVL occurrence as their postoperative effect. For each patient, vital data, collected every 4 seconds, consists of heart rate (HR), mean arterial pressure (MAP), right atrium pressure (RAP), oxygen saturation $\left(\mathrm{SpO}_{2}\right)$, hemoglobin $(\mathrm{Hb})$, hemoglobin oxygen content $\left(\mathrm{HbO}_{2}\right)$ and relative cerebral blood flow (rCBF). $\mathrm{Hb}, \mathrm{HbO}_{2}$ and $\mathrm{rCBF}$ are collected using near-infrared spectroscopy (NIRS). The NIRS is a 
spectroscopic method that uses the near-infrared region of the electromagnetic spectrum. Demographic data collected includes sex, type of disease, cardiac bypass duration and deep hypothermic circulatory arrest duration for each patient as well as blood gas measurements. Collected blood gas measurements are presented in Tab. I.

TABLE I

Collected Blood Gas Data

\begin{tabular}{ccc}
\hline Measurement & Description & Unit \\
\hline $\mathrm{pH}$ & Arterial blood $\mathrm{pH}$ & \\
$\mathrm{PaCO}_{2}$ & Partial pressure of dissolved $\mathrm{CO}_{2}$ & $\mathrm{mmHg}$ \\
$\mathrm{PaO}_{2}$ & Partial pressure of dissolved $\mathrm{O}_{2}$ & $\mathrm{mmHg}$ \\
$\mathrm{HCO}_{3}$ & Concentration of bicarbonate ions & $\mathrm{mmol} / \mathrm{L}$ \\
$\mathrm{O}_{2} \mathrm{Sat}$ & Arterial oxygen saturation & $\%$ \\
$\mathrm{Hgb}$ & Hemoglobin concentration & $\mathrm{g} / \mathrm{dL}$ \\
$\mathrm{K}^{+}$ & Ionized Potassium & $\mathrm{mmol} / \mathrm{L}$ \\
$\mathrm{Ca}^{++}$ & Ionized Calcium & $\mathrm{mmol} / \mathrm{L}$ \\
$\mathrm{Na}^{+}$ & Ionized Sodium & $\mathrm{mmol} / \mathrm{L}$ \\
$\mathrm{Hct}$ & Hematocrit & $\%$ \\
\hline
\end{tabular}

In our previous work [19] we developed a cycle-averaged model of the HLHS heart to study effects of the various parameters on surgical outcome. That model provides a very useful tool to better understand the principal factors that drive the HLHS physiology and could be significant for improving patient management. However, there is no physics based model for the blood gas data and also of vital data such as hemoglobin which renders application of Computational Intelliegence based techniques particularly attractive for this problem.

\section{B. Feature Extraction}

Based on the collected set of physiological parameters a feature pool was developed. The feature pool is different for vital and blood gas measurements. The derived features of vital measurements include: minimum, maximum, mean, variation, skewness, kurtosis and trend. Skewness and kurtosis are third and forth order statistical moments of a random variable defined by Eq. (1).

$$
m_{n}(x)=E\left\{(x-\mu)^{n}\right\}
$$

where, $n$ is the order, $\mu$ is the mean value of the data and $E$ is the expected value. The derived features from the blood gas data are maximum and minimum values of the rate of change of measurements, time weighted mean, and out of range index (ORI). The rate of change of blood gas measurement is defined as the slope of the line connecting two consecutive measurements. Blood gas measurements are discontinuous and to overcome this problem the data is linearly interpolated assuming there is no sudden change happening in the data, a fact confirmed by the clinicians. Time weighted mean is simply calculated using Eq. (2).

$$
M_{w}(x)=\frac{\sum_{i=1}^{m} t_{i} \times x_{i}}{\sum_{i=1}^{m} t_{i}}
$$

where, $m$ is the number of measurements and $x$ is the measured variable. We define the out of range index (ORI) as an area which is bounded by lower or upper normal range of the data and the measurement waveform. The upper and lower limit of the normal range for the measured blood gas data are presented in Tab. II. This index is an important indicator taking into account both the time that variable has been out of range and also the out of range value. The ORI has the unit of the variable it is calculated for multiplied by time. For example the ORI for $\mathrm{PaCO}_{2}$ has units of mmhg.s. Figure (1) shows the defined feature for a data sample.

TABLE II

Normal Range of Blood Gas Data

\begin{tabular}{ccc}
\hline Measurement & Lower Limit & Upper Limit \\
\hline$p H$ & 7.34 & 7.44 \\
$\mathrm{PaCO}_{2}$ & 35 & 45 \\
$\mathrm{PaO}_{2}$ & 75 & 100 \\
$\mathrm{HCO}_{3}$ & 22 & 26 \\
$\mathrm{O}_{2} \mathrm{Sat}$ & 95 & 100 \\
$\mathrm{Hgb}$ & 14 & 16 \\
$\mathrm{~K}^{+}$ & 3.5 & 5 \\
$\mathrm{Ca}^{++}$ & 8.5 & 10.5 \\
$\mathrm{Na}$ & 135 & 145 \\
$\mathrm{Hct}$ & 36 & 44 \\
\hline
\end{tabular}

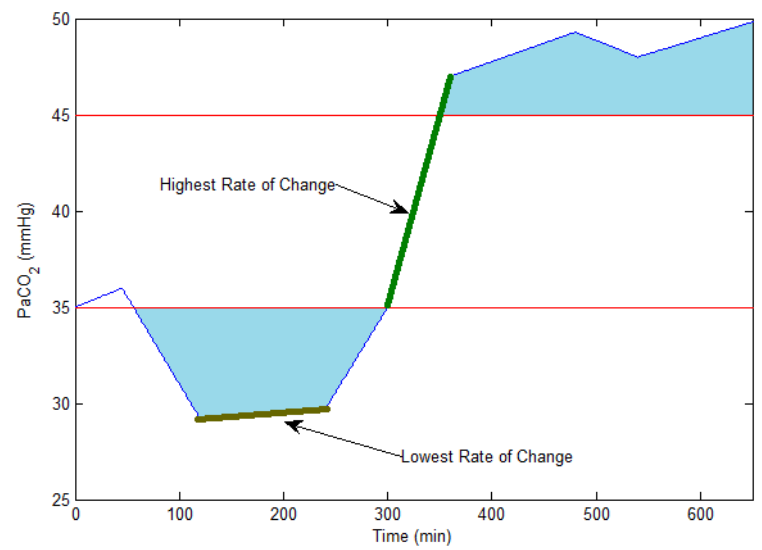

Fig. 1. Plot of features derived from a sample blood gas measurement The blue area is the ORI index of the $\mathrm{PaCO}_{2}$ for a sample patient.

\section{DECISION TREE}

The goal of building a DT is to find classification rules from the input data. The DT consists of a root, nodes and leaves. Leaves are terminal nodes and each leaf represents a class. There are two phases in DT construction: tree building and tree pruning.

\section{A. Tree Building}

The CART algorithm [20], which uses the recursive partitioning approach to DT rule induction, is employed to develop DT. The algorithm selects a criterion to split the data and build the tree. To split the data, DT maximizes the information gain (IG) of the output data (which in turn 
reduces the information entropy). The IG of an attribute $A$ is used to select the best splitting criterion attribute. The features with the highest IG is then selected to build the DT.

A large number of different values for the output will cause problems in implementing the information gain measure of the data; hence in this case, the gain ratio is used instead [16]. The Gini index (GI) is an impurity-based criterion that measures the divergence between the probability distributions of the target attributes values [21]. The GI is defined by Eqn. (3) and Eqn. (4) as follows.

$$
G I(D)=G(D)-\sum_{j=1}^{v} p_{i} G\left(D_{j}\right)
$$

where,

$$
G(D)=1-\sum_{i=1}^{m} p_{i}^{2}
$$

where, $m$ is the number of classes (the classes in this study are PVL and health), $p_{i}$ is the probability of class $i$ in dataset $D,\left|D_{j}\right|$ is the number of observations with attribute value $j$ in dataset $D,|D|$ is the total number of observations in dataset $D$, and $v$ is number of all attribute values. For example, if attribute $S$ has values $\{1,2,2,3,2,3,1\}$, then $v$ will be 3 .

\section{B. Tree Pruning}

The constructed tree in the building phase is usually complex, and tends to be a over-fit to the training data. To overcome overfitting and improve generalization of the developed classification tree, we prune the tree using a Fishers exact test (FET) based pruning approach as described in [14], [22]. We use 0.05 as the $p$-value threshold for pruning the selected node.

\section{RESULTS}

A DT based on the features derived from blood gas data was generated and is shown in Fig. (2). Results show that among all the variables and features the maximum rate of change of $\mathrm{HCO}_{3}$ and the minimum rate of change of $\mathrm{PaCO}_{2}$ are the most important parameters for the PVL occurrence prediction. This result confirms our previous findings presented in [17] which highlighted the role of blood $\mathrm{CO}_{2}$ concentration as an important factor in the PVL prediction; that study was based on a more primitive dataset. This result also shows the importance of the rate of change in blood gas data as an indicator of hemodynamic instability which could lead to PVL. The developed DT also shows that the following rule plays the strongest role in the prediction of PVL based on the blood gas measurements.

$$
\begin{array}{cl}
\text { if } \quad\left(R C_{\text {max }}\left(\mathrm{HCO}_{3}\right)>0.04 \wedge R C_{\text {min }}\left(\mathrm{PaCO}_{2}\right)>-0.17\right) \\
\text { then } \quad(\text { Prediction : } \mathrm{PVL})
\end{array}
$$

where, $\wedge$ is the logical and operator.

In order to investigate the next important features of blood gas measurements, we drop rate of change features from our feature pool and form a DT based on the remaining feature pool. The resulting DT is shown in Fig. (3) and illustrates the importance of our defined ORI. The results are surprising

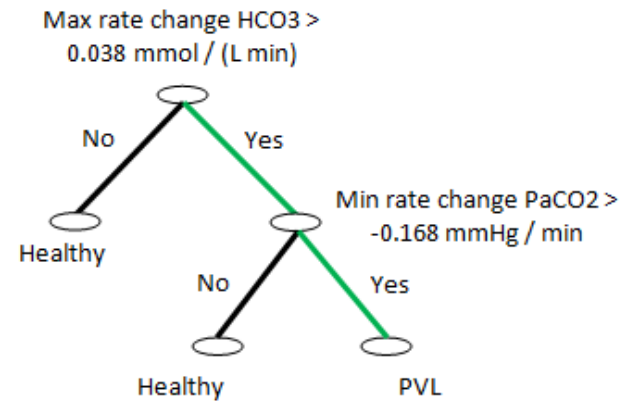

Fig. 2. Result of forming DT for all features derived from blood gas data. Results show that among all variables and features maximum rate of change of $\mathrm{HCO}_{3}$ and minimum rate of change of $\mathrm{PaCO}_{2}$ are the most valuable parameters for predicting PVL. The green path represent the strongest rule in DT decision making.

in that the DT shows that an increase in $\mathrm{Ca}^{++}$and $\mathrm{K}^{+}$ ORI and the condition that $\mathrm{HCO}_{3}$ ORI is high will decrease the probability of PVL occurrence. At this time the exact physiological basis for this finding is not understood.

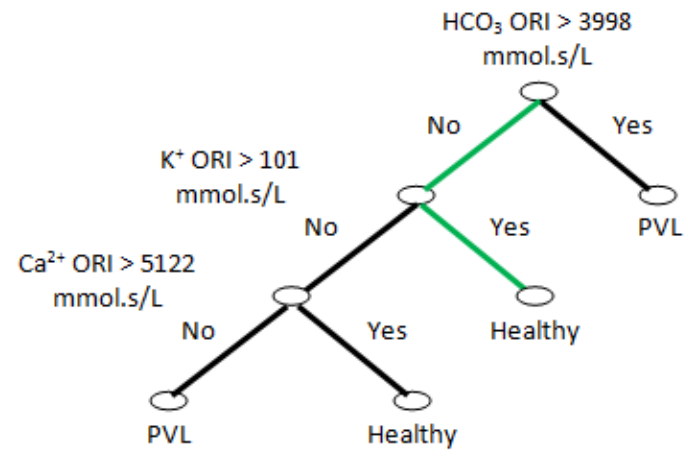

Fig. 3. Result of forming DT from remaining features derived from blood gas data. Results show that $\mathrm{K}, \mathrm{CA}$ and $\mathrm{HCO}_{3}$ ORI are the most valuable parameters for predicting PVL. The green path represent the strongest rule in DT decision making.

Next, a DT is built from the features of vital measurements and is shown in Figure (4). According to this, the kurtosis of $\mathrm{HR}$ and $\mathrm{Hb}$ are the most important factors for PVL prediction. In probability theory and statistics, kurtosis is any measure of the "peakedness" of the probability distribution of a real-valued random variable [23]. High kurtosis is associated with more outlying values. Increased HR uncertainty is suggested as a PVL predictor in some references [4]. A possible explanation for this result is that an increase in uncertainty in HR will increase instability in the cardiac output which directly affects the oxygen delivery to the brain. Insufficient and unstable supply of oxygen will severely damage any organ, especially a sensitive organ such as the brain.

\section{CONCLUSIONS}

Periventricular leukomalacia or PVL is a type of white matter brain injury which is common in neonates after con- 


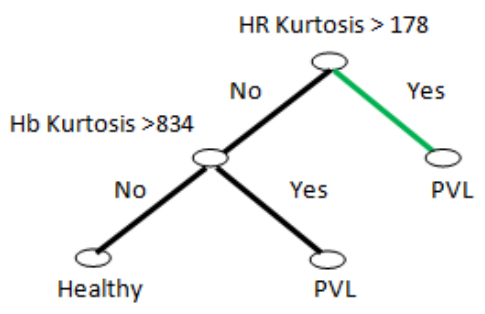

Fig. 4. Result of forming DT from features derived from vital measurements. Results show that kurtosis of $\mathrm{HR}$ and $\mathrm{Hb}$ are the most important factors for PVL prediction from vital measurements. The green path represents the strongest rule in DT decision making.

genital heart surgery. The exact causes of PVL still remain unknown. In this paper we have applied a Computational Intelligence technique to discover and highlight hidden patterns in the hemodynamic data collected from neonates after heart surgery which may aid in the development of a predictive tool for this debilitating condition. Our results show that $\mathrm{HR}$ and $\mathrm{Hb}$ from vital data are the most important vital measurements to consider for the PVL prediction. Moreover, the maximum and minimum values of the rate of change of $\mathrm{HCO}_{3}$ and $\mathrm{PaCO}_{2}$ are the most important parameters from the blood gas data. While the findings of this study seem to be very interesting and important, it still needs more validation from a physiological point of view. Hence, the next step of the study is to investigate the physiological reasons behind these findings. For example, questions that need investigating include: how does increased uncertainty in $\mathrm{Hb}$ result in the PVL occurrence and how does the $\mathrm{CO}_{2}$ affect the PVL occurrence. Furthermore, additional data is needed to prove the robustness of the developed algorithm to measurement noise and application to special cases.

\section{ACKNOWLEDGMENT}

The research reported in this paper is supported by a grant from National Institute of Health (No. 1 R01 NS 72338 01A1).

\section{REFERENCES}

[1] K. K. Galli, R. A. Zimmerman, G. P. Jarvik, G. Wernovsky, M. K. Kuypers, R. R. Clancy, L. M. Montenegro, W. T. Mahle, M. F. Newman, A. M. Saunders, S. C. Nicolson, T. L. Spray, and J. W. Gaynor, "Periventricular leukomalacia is common after neonatal cardiac surgery," J Thoracic Cardiovascular Surgery, vol. 127, no. 3, pp. 692-704, Mar 2004.

[2] J. J. Volpe, Neurology of the Newborn, 4th ed. Saunders, 2001.

[3] — - "Cerebral white matter injury of the premature infant-more common than you think," Pediatrics, vol. 112, pp. 176-180, 2003.

[4] J. W. Gaynor, "Periventricular leukomalacia following neonatal and infant cardiac surgery," Seminars in Thoracic and Cardiovascular Surgery: Pediatric Cardiac Surgery Annual, vol. 7, pp. 133-140, 2004.

[5] P. S. McQuillen, D. A. Goff, and D. J. Licht, "Effects of congenital heart disease on brain development," Progress in Pediatric Cardiology, vol. 29, no. 2, pp. 79-85, 2010.

[6] P. S. McQuillen and S. P. Miller, "Congenital heart disease and brain development," Annals of the New York Academy of Sciences, vol. 1184, pp. 68-86, 2010.
[7] H. C. Glass, S. Fujimoto, C. Ceppi-Cozzio, A. I. Bartha, D. B Vigneron, A. J. Barkovich, D. V. Glidden, D. M. Ferriero, and S. P. Miller, "White-matter injury is associated with impaired gaze in premature infants," Pediatric Neurololgy, vol. 38, no. 1, pp. 10-15, 2008.

[8] I. C. van Haastert, L. S. de Vries, M. J. C. Eijsermans, M. J. Jongmans, P. J. M. Helders, and J. W. Gorter, "Gross motor functional abilities in preterm-born children with cerebral palsy due to periventricular leukomalacia," Developmental Medicine and Child Neurology, vol. 50, no. 9, pp. 684-689, Sep 2008.

[9] A. Gururaj, L. Sztriha, A. Bener, A. Dawodu, and V. Eapen, "Epilepsy in children with cerebral palsy," Seizure: the Journal of the British Epilepsy Association, vol. 12, pp. 110-114, 2003.

[10] V. Podgorelec, P. Kokol, and M. M. Stiglic, "Searching for new patterns in cardiovascular data," in Proc. 15th IEEE Symp. ComputerBased Medical Systems (CBMS 2002), 2002, pp. 111-116.

[11] K. C. Tan, Q. Yu, C. M. Heng, and T. H. Lee, "Evolutionary computing for knowledge discovery in medical diagnosis," Artificial Intelligence in Medicine, vol. 27, no. 2, pp. 129-154, 2003.

[12] A. C. Stasis, E. N. Loukis, S. A. Pavlopoulos, and D. Koutsouris, "A decision tree-based method, using auscultation findings, for the differential diagnosis of aortic stenosis from mitral regurgitation," in Proc. Computers in Cardiology, 2003, pp. 769-772.

[13] G. Dounias and D. Linkens, "Adaptive systems and hybrid computational intelligence in medicine," Artificial Intelligence in Medicine, vol. 32, no. 3, pp. 151-155, Nov 2004.

[14] A. Singh and J. V. Guttag, "A comparison of non-symmetric entropybased classification trees and support vector machine for cardiovascular risk stratification," in Proc. Annual Int Engineering in Medicine and Biology Society, EMBC Conf. of the IEEE, 2011, pp. 79-82.

[15] B. Samanta, G. L. Bird, M. Kuijpers, R. A. Zimmerman, G. P Jarvik, G. Wernovsky, R. R. Clancy, D. J. Licht, J. W. Gaynor, and C. Nataraj, "Prediction of periventricular leukomalacia. part I: Selection of hemodynamic features using logistic regression and decision tree algorithms," Artificial Intelligence in Medicine, vol. 46, no. 3, pp. 201-215, 2009.

[16] M. A. Karaolis, J. A. Moutiris, D. Hadjipanayi, and C. S. Pattichis, "Assessment of the risk factors of coronary heart events based on data mining with decision trees," IEEE Transactions on Information Technology in Biomedicine, vol. 14, no. 3, pp. 559-566, 2010.

[17] B. Samanta, G. L. Bird, M. Kuijpers, R. A. Zimmerman, G. P. Jarvik, G. Wernovsky, R. R. Clancy, D. J. Licht, J. W. Gaynor, and C. Nataraj, "Prediction of periventricular leukomalacia. part II: Selection of hemodynamic features using computational intelligence," Artificial Intelligence in Medicine, vol. 46, no. 3, pp. 217-231, 2009.

[18] A. Jalali, A. Ghaffari, P. Ghorbanian, and C. Nataraj, "Identification of sympathetic and parasympathetic nerves function in cardiovascular regulation using ANFIS approximation," Artificial Intelligence in Medicine, vol. 52, no. 1, pp. 27-32, 2011.

[19] A. Jalali and C. Nataraj, "A cycle-averaged model of hypoplastic left heart syndrome (HLHS)," in Proc. Annual Int Engineering in Medicine and Biology Society, EMBC Conf. of the IEEE, 2011, pp. 190-194.

[20] J. R. Quinlan, C4.5: Programs for machine learning. San Francisco, CA, USA: Morgan Kaufmann Publishers Inc., 1993.

[21] L. Breiman, J. Friedman, C. J. Stone, and R. A. Olshen, Classification and Regression Trees. Wadsworth Int. Group, 1984.

[22] W. Liu, S. Chawla, D. Cieslak, and N. C. A, "A robust decision tree algorithms for imbalanced data sets," in Proceedings of the Tenth SIAM International Conference on Data Mining. Society for Industrial and Applied Mathematics, 2010, pp. 766-777.

[23] Y. Dodge, D. Cox, D. Commenges, A. Davison, P. Solomon, an S. Wilson, Eds., The Oxford Dictionary of Statistical Terms, 6th ed. Oxford University Press, 2006. 